Author(s):

Submitted to:
J. Schultz, CST-1

S. Czuchlewski, CST-1

R. Karl, CST-1

T. Myer, DoD-PO

G. Nickel, P-22

W. Buttler, Univ. of TX

T. Carrig, Cornell Univ.

N. Cockroft, Univ. of Canterbury

E. Eloranta, Univ. of WI

R. Marchbanks, UNM

K. Moore, UCLA

R. Petrin, OK State Univ.

DOE Office of Scientific and Technical Information (OSTI)

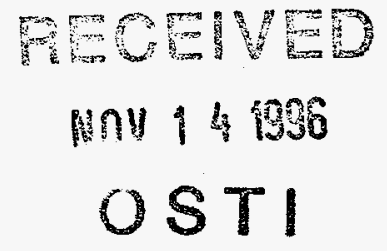

RECEIVED

mก 1936

OSTI

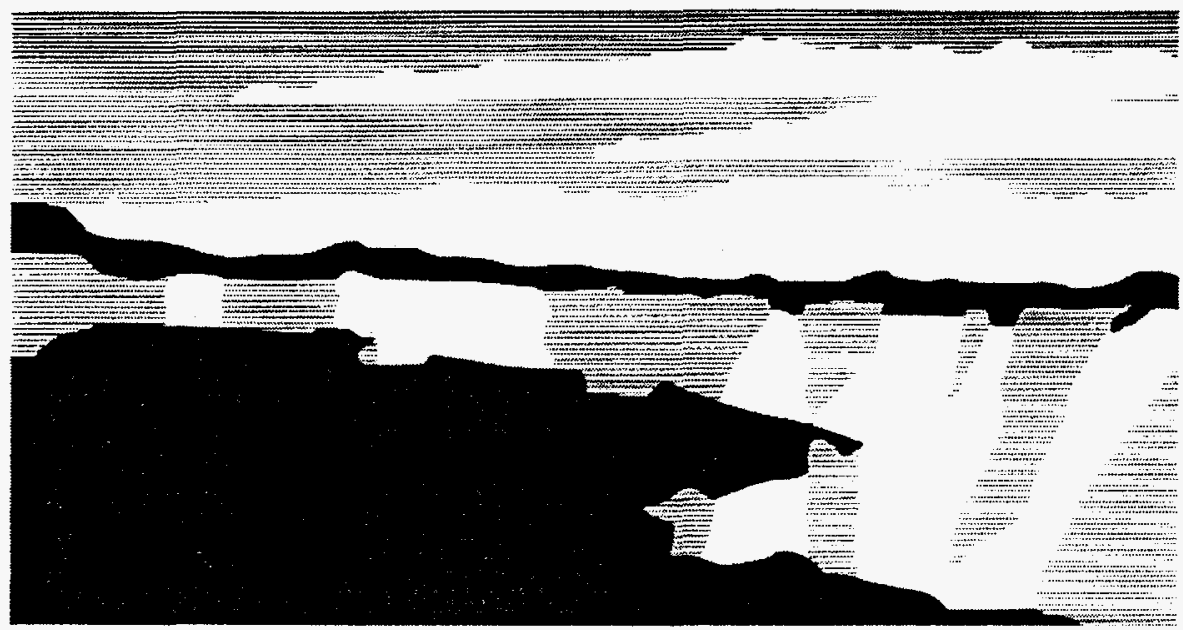

Los Alamos National Laboratory, an affirmative action/equal oppontunity employer, is operated by the University of California for the U.S. Department of Energy under contract W-7405-ENG-36. By acceptance of this article, the publisher recognizes that the U.S. Government retains a nonexclusive, royaltyfree license to publish or reproduce the published form of this contribution, or to allow others to do so, for U.S. Government purposes. The Los Alamos National Laboratory requests that the publisher identify this article as work performed under the auspices of the U.S. Department of Energy. 


\section{DISCLAIMER}

This report was prepared as an account of work sponsored by an agency of the United States Government. Neither the United States Government nor any agency thereof, nor any of their employees, makes any warranty, express or implied, or assumes any legal liability or responsibility for the accuracy, completeness, or usefulness of any information, apparatus, product, or process disclosed, or represents that its use would not infringe privately owned rights. Reference herein to any specific commercial product, process, or service by trade name, trademark, manufacturer, or otherwise does not necessarily constitute or imply its endorsement, recommendation, or favoring by the United States Government or any agency thereof. The views and opinions of authors expressed herein do not necessarily state or reflect those of the United States Government or any agency thereof. 


\section{DISCLAIMER}

Portions of this document may be illegible in electronic image products. Images are produced from the best available original document. 


\title{
Advanced Laser Remote Sensing
}

J. Schultz*, S. Czuchlewski, R. Karl, T. Meyer, and G. Nickel, Los Alamos National Laboratory; W. Buttler, University of Texas - Austin; T. Carrig, Cornell University; N. Cockroft, Univ. of Canterbury; E. Eloranta, University of Wisconsin - Madison; R. Marchbanks, University of New Mexico; K. Moore, UCLA; R. Petrin, Oklahoma State University

\begin{abstract}
This is the final report of a three-year, Laboratory-Directed Research and Development (LDRD) project at the Los Alamos National Laboratory. Remote measurement of wind velocities is critical to a wide variety of applications such as environmental studies, weather prediction, aircraft safety, the accuracy of projectiles, bombs, parachute drops, prediction of the dispersal of chemical and biological warfare agents, and the debris from nuclear explosions. Major programs to develop remote sensors for these applications currently exist in the DoD and NASA. At present, however, there are no real-time, three-dimensional wind measurement techniques that are practical for many of these applications and we report on two new promising techniques. The first new technique uses an elastic backscatter lidar to track aerosol patterns in the atmosphere and to calculate three dimensional wind velocities from changes in the positions of the aerosol patterns. This was first done by Professor Ed Eloranta of the University of Wisconsin using post processing techniques and we are adapting Professor Eloranta's algorithms to a real-time data processor and installing it in an existing elastic backscatter lidar system at Los Alamos (the XM94 helicopter lidar), which has a compatible data processing and control system. The second novel wind sensing technique is based on radio-frequency (RF) modulation and spatial filtering of elastic backscatter lidars. Because of their compactness and reliability, solid state lasers are the lasers of choice for many remote sensing applications, including wind sensing. At present, however, there are no suitable solid state lasers that operate in the 1.6 to 2.0 micron
\end{abstract}

*Principal investigator, e-mail: jschultz@lanl.gov 
wavelength band, which is critical to many laser detection and ranging (lidar) remote sensing applications because laser light at these wavelengths is eyesafe, detectable with room temperature detectors, and short enough in wavelength to be scattered efficiently by atmospheric aerosols and particulates. Our eyesafe laser development efforts are focused on use of the erbium $3+$ ion in various crystalline hosts to generate two laser wavelengths simultaneously by means of a novel cascade lasing scheme in which lasing at 2.9 microns maximizes laser power at the desired 1.6 micron wavelength.

\section{Background and Research Objectives}

To date, most lidar wind sensing programs have focused on heterodyne Doppler lidar systems, which are adequate for some applications but are limited by a trade-off between the scattering cross sections of atmospheric aerosols and the high opto-mechanical tolerances that optical heterodyne receivers require. At long wavelengths, the tolerances are less stringent, but scattering cross sections are low, while at shorter wavelengths the scattering cross sections are higher but the tolerances are very difficult to achieve. In addition, Doppler techniques are limited in their ability to measure cross winds and cross winds are the wind components of greatest importance for many applications.

At present, there are no real-time, three dimensional wind measurement techniques that are practical for many applications and so two promising novel techniques were investigated. The first new technique uses an elastic backscatter lidar and advanced signal processing techniques to identify and track aerosol patterns in the atmosphere and to calculate three-dimensional wind velocities from changes in the positions of the aerosol patterns. This was first done by Professor Ed Eloranta of the University of Wisconsin using post processing techniques. Under this LDRD project, Professor Eloranta's algorithms are being adapted to real-time processing of the types of data sets that may be taken from moving, operational platforms. The second technique is based on radiofrequency $(\mathrm{RF})$ modulation and spatial filtering of elastic backscatter lidar return signals. Both of these efforts were begun with the objective of evaluating their potential for future applications and developing sufficient expertise to test the technology for those applications.

One of the major obstacles to widespread use of lidar technology for military and civilian applications is the potential for exposing personnel to harmful levels of laser light. Because eye injury thresholds are approximately three orders of magnitude higher at wavelengths longer than about 1.5 microns and because it is possible to use high speed, 
room temperature APD detectors at wavelengths shorter than about 1.7 microns, the wavelength range of choice for a wide variety of lidar applications lies between 1.5 and 1.7 microns. Due to their compactness, ruggedness, and inherent reliability, solid-state lasers are the lasers of choice for many applications, but unfortunately, there is no conventional solid-state laser that operates efficiently between 1.5 and 1.7 microns. The second objective of this project was therefore evaluation and development of new, solid-state eyesafe lasers for lidar applications.

The third objective of this project was to study the feasibility of using lidar to measure atmospheric parameters such as winds, humidity, and temperatures which are related to the transport of airborne materials within the planetary boundary layer. This is related to NASA's long-standing interest in global meteorology, but many applications of interest to Los Alamos are regional or local in scale, with significantly different measurement requirements. A study of the measurement requirements, potential operational platforms, and lidar techniques and technologies was initiated and opportunities for applications were explored.

\section{Importance to Los Alamos' Science and Technology Base and National R\&D Needs}

Lidar research is a rapidly growing field with myriad civilian and national security applications. A few examples that involve wind sensing and are pertinent to this project include environmental studies; weather prediction; aircraft safety; the accuracy of projectiles, bombs, and parachute drops; predicting the dispersal of effluents from the production of weapons of mass destruction, chemical, and biological warfare agents on the battlefield, and debris from nuclear explosions and attacks on production facilities. For some of these applications, lidar is an operational tool and the development of improved lidar techniques and technologies is the focus of the research; for other applications, lidar is a critical tool used in support of other research objectives. In either case, improved competence in advanced lidar technology is important to maintaining and enhancing Los Alamos' science, technology, and programmatic base. At present, lidar projects are bringing approximately $\$ 15 \mathrm{M}$ to $\$ 20 \mathrm{M}$ per year in external funding to Los Alamos, and this LDRD project will expand both the scope and the magnitude of the Laboratory's involvement in lidar and other research.

Within the national security sector, national needs for detecting and countering proliferation activities are the primary focus of this project. Wind sensing is of interest to counterproliferation because it allows accurate prediction of chemical and biological agent 
dispersal on the battlefield as well as prediction of the dispersal of the debris from strikes against facilities that are producing weapons of mass destruction. Wind (and perhaps water vapor and temperature) sensing can also contribute to proliferation detection by improving the positioning and interpretation of sample collection devices and point sensors.

Within the civilian sector, wind measurement is of great interest to NASA's Earth Observing System program and has already been the subject of major investments. Microand meso-scale meteorology is of great interest to a wide variety of environmental, biological, and agricultural research activities and when used in conjunction with lidar systems, may be a key to full utility of lidar systems for the proliferation detection and counterproliferation applications listed above.

Realization of operational lidars for many of the applications mentioned above will benefit from the development of eye-safe lasers due to the potential for exposing personnel to laser light. Eye safety and system reliability are the greatest concerns in any military application of lasers for things such as target designators, range finders, survey instruments, etc., and our project addresses both issues. As an example of this, USA CBDCOM has stated that the development of eyesafe lasers is one of its highest research priorities and eyesafe lasers will be used by Los Alamos' Counter-Proliferation Lidar (CP Lidar) Project.

\section{Scientific Approach and Results}

In this project there was significant early work towards the development of several new sensor/electronics technologies including the application of high-density-interconnect multi-chip-modules (HDI-MCM) as sensors and microprocessors; two new sensor technologies--LENA, a low energy neutral atom imager, and NIC, a near-infrared camera; and the investigation of erbium-doped YAG as an eye-safe laser (ESL) for lidar applications.

\section{A. High-Density-Interconnect Multi-Chip-Modules}

High-density-interconnect multi-chip-modules (HDI-MCM) can best be described as the integration of sensor devices, signal-processing capabilities, and mechanical packaging to provide enhanced sensor sensitivity, higher bandwidth and intelligence within a densely interconnected, rugged substrate. Multiple sensor capabilities can be combined with specialized ASICs to provide sensor systems that are much smaller, consume orders of magnitude less power, and are tens to hundreds of times faster than today's technology. 
We are investigating the fabrication of HDI-MCMs using sensor materials of interest with specially designed ASICs for signal processing to understand the potential use of such packages as advanced sensor systems. An embedded sensor test system was fabricated that had custom ASICs and mounting pads for specific sensors. The circuitry is an eightchannel, analog, peak-detector front end, with an adjustable eight-channel threshold circuit, which feeds the logic circuit. An MCM processor package that includes an R3081, 25MIP, 5-MOP central processor, 4 Mbytes of SRAM, 1 Mbyte of EEPROM, and a high speed interconnect bus was also designed and fabricated. This MCM is the equivalent of a desk-top workstation in a 1.25 " x 1.25 " $\mathrm{x} .01$ " package.

\section{B . Near Infrared Camera (NIC)}

A critical aspect of mesospheric dynamics and energy balance is the contribution of breaking and dissipating gravity waves. These waves can be studied by direct imaging of the wave-modulated $\mathrm{O} 2(0,0)$ airglow layer at approximately $90 \mathrm{~km}$ altitude. Indeed, similar wave measurements are now routinely performed from the ground and have been demonstrated, but not yet exploited, from orbit. A mission of NISSE, the proposed Norwegian small satellite project, is to perform an orbital global survey of atmospheric gravity waves through measurements of airglow modulation. The scientific merit of the mission is based in refining the understanding of the latitudinal, geographical and seasonal variation of gravity waves as they relate to wave coupling in the atmosphere. These investigations are viewed to be important because of the recognized need for improved knowledge of wave source locations and tidal wind filtering of gravity waves on a global basis, which would result in improved atmospheric circulation modeling.

The survey of atmospheric waves by NISSE is supported by the Near Infrared Camera (NIC) experiment. From the proposed NIC measurements, a database of observations will be constructed that categorize airglow intensity and gravity wave contrast as a function of horizontal wavelength, coherence length, time of observation, and azimuth of phase fronts. These data entries can then be sorted by occurrence over latitude and longitude, or other spatial and temporal projections, to generate a spectral map of intensities, wavelengths, and propagation directions. Hence, constraints on source distribution and gravity wave filtering in the atmosphere below airglow heights will be established. NIC activities have included: defining the NIC experiment and ordering a CCD camera for a ground based station for taking comparative measurements; setting up the ground-based experiment station; and evaluating near-infrared imaging and system performance constraints through execution of a ground-based wave-imaging campaign. 


\section{Superconducting Tunneling Junctions}

In superconducting tunneling junction detectors, incoming photons or particles break Cooper pairs. The resulting quasiparticles tunnel through the junction to give the measured

detector current. The binding energy of Cooper pairs is the order of $10^{-3} \mathrm{eV}$ whereas, the binding energy of electron-hole pairs in silicon is the order of $1 \mathrm{eV}$. This much smaller band gap extends the range of STJs down into the IR and gives them much better energy resolution than seen in silicon detectors. The number of quanta in an STJ is about 1000 times larger than that in standard silicon detectors. The contribution to the resolution by statistical uncertainty is then approximately 0.03 times smaller. In addition to the high energy and spatial resolution, the STJ detector has a short response time (count rates up to $10^{7}$ photons/sec are possible) and works from infrared through optical and UV up to $10 \mathrm{~s}$ of keVs. There are several challenges that need to be overcome for STJ detectors to be viable detectors in space. They operate at temperatures less than $1^{\circ} \mathrm{K}$ and have small signals that are subject to degradation by electronic noise. This year we have established a facility to test STJ detectors and have started development of the detectors themselves.

HDI-MCM can best be described as the integration of sensor devices, signalprocessing capabilities, and mechanical packaging to provide enhanced sensor sensitivity, higher bandwidth and intelligence within a densely interconnected rugged substrate. Multiple sensor capabilities can be combined with specialized ASICs to provide sensor systems that are much smaller, consume orders of magnitude less power, and are tens to hundreds of times faster than today's technology. We investigated the fabrication of HDIMCMs using sensor materials of interest with specially designed ASICs for signal processing to understand the potential use of such packages as advanced sensor systems. The focus will be on developing a suitable circuit/systems approach that maximizes the performance of the sensor package. We have completed the design and layout of an initial HDI-MCM sensor/electronic component and addressed the packaging issues. We have also developed advanced analytical tools to model the thermal and electromagnetic environment of the components.

\section{Data Analysis and and Assessment Computer (DAAC)}

Several issues associated with advanced sensors and small spacecraft include storage of raw ("level 0") instrument data, development of algorithms to process the data, routine processing of raw data using these algorithms to generate "level 1" and "level 2" data, archival of these data products, and making the data products easily available to users. Los 
Alamos has extensive experience with these issues on existing satellite projects and this expertise can be applied to remote sensing. With respect to the Earth Observing System Data Information System (EOSDIS), NASA is addressing these same issues with the DAAC facilities. The Los Alamos DAAC, will be based on the Version 0 architecture, and is fully compatible with the existing NASA DAACs. This DAAC will functionally support research into short- and medium-term environmental characterization and ecological issues that directly affect policy decisions by the government. It will also make available to the broader user community existing and future data sets from Los Alamos instrumentation applicable to questions of global climate change. We have worked with other facilities at Marshall, Goddard, and Sioux Falls. Two mass storage systems, one capable of 8-10 TeraBytes long-term storage and the other 100-200 GigaBytes fast access were purchased. Initial software for the DAAC operation was written and several different data storage formats developed.

\section{E. Aerosol-Drift Wind Sensing for Tactical Battlefield Applications}

Initial evaluation of the aerosol-drift and RF-modulation/spatial-filtering wind-sensing techniques indicated that the aerosol-drift technique is reasonably mature and promising. Significant effort was therefore devoted to the aerosol-drift technique. Algorithms developed at the University of Wisconsin during the last decade were modified and simple processing codes were written in the Mathcad and $\mathrm{C}$ languages. Data sets were generated both artificially and experimentally using the XM-94 helicopter lidar for use with those processing codes. The experimental data sets were used to conduct speed tests with the codes written in $\mathrm{C}$ and refinements to the algorithms and codes were developed. Contact was also established with Professor Ed Eloranta of the University Wisconsin - Madison (UW-M), who developed the original algorithms.

Based on this exploratory work, it was determined that detailed development of threedimensional, real-time algorithms that are compatible with operational platforms could be most efficiently accomplished through an active collaboration with Professor Eloranta. A contract with UW-M was therefore established to do joint development of the algorithms, use an existing UW-M lidar to take sample data sets compatible with moving operational platforms, and use the data sets to test and refine the algorithms. Professor Eloranta has developed initial versions of the new algorithms and taken several data sets with his lidar. 


\section{F. Use of Lidar in Predicting Transport of Effluents from Proliferation Activities}

At present, much of the United States' operational proliferation detection capability depends on sampling detectors that must be positioned in the effluent streams of suspected proliferation facilities. To date, positioning has been based on meso-scale meteorological modeling and fragmentary (if any) weather data, which reduces the effectiveness of deployed assets and the certainty with which collected samples can be attributed to specific sites.

At the outset of the study, access to proliferation sites, potential lidar platforms, and probable measurement requirements were considered to determine the constraints on the problem. Due to a combination of peacetime restrictions on airspace violation and probable needs for a measurement grid covering hundreds of square kilometers with frequent revisit times, it was determined that satellites are the most promising platform. However, wind patterns are strongly dependent on the time of day, particularly in complex terrain and along coastlines. Since satellite revisit times are generally between twenty and forty hours, which is much longer than the time scale of daily changes in wind patterns, it became apparent that the measured data may have to be used in conjunction with mesoscale modeling for greatest utility. (This is exciting science because large grids of meteorological data have never been available before, and rapid advances in the understanding of mesometeorology are likely to follow.)

The next step in the study was reading of the extensive literature on meteorological applications of lidar to determine probable constraints on the number of measurements that can be made around a specific site in a satellite pass and to select candidate lidar techniques. The aerosol-drift wind-measurement technique was rejected because too much laser energy is required to make a single wind measurement from space, which would result in too sparse a grid to be very useful. If used at $\mathrm{CO}_{2}$ laser wavelengths of approximately 10 microns, the heterodyne or "coherent" doppler wind-measurement technique would probably provide the necessary wind measurements, but this wavelength range is not suitable for water vapor or temperature measurements, which may also be required for this application. Based on these considerations, "incoherent" doppler wind-measurement techniques that measure the doppler shift with edge filters or Fabry-Perot interferometers at laser wavelengths between 0.3 and 1.6 microns were chosen as the top candidates for further development. A report on the study is being written. 
During the course of this study contacts were established with the extensive NASA/NOAA meteorological lidar community and with researchers at the University of Michigan. Opportunities for joint efforts were discussed and contact was made between the appropriate DOE and NASA managers. Los Alamos personnel attended meetings of the NOAA Working Group on Space-Based Lidar Winds in January and July of 1995. A lifecycle plan for the development of meteorological lidars in support of proliferation detection was written and submitted to DOE/NN-20.

During a visit to NASA's Langley Research Center, common interest was identified in $\mathrm{Cr}$ :LiSAF lasers based on their potential suitability for lidar measurement of a wide variety of atmospheric parameters. To primarily demonstrate the capabilities of Cr:LiSAF laser technology, a joint Cr:LiSAF laser demonstration was executed at Los Alamos in August of 1995. The laser hardware used for this demonstration was drawn from both organizations and assembled by a joint team and the specific configuration used is traceable to configurations useful for operational lidar systems. Several performance records for $\mathrm{Cr}: \mathrm{LiSAF}$ lasers were established and at the conclusion of the effort the laser system was installed in a Los Alamos lidar system (the "white truck"). Some water vapor measurements were made, but the laser diodes used to seed the Cr:LiSAF oscillator (thus controlling the lasing wavelength) failed during the lidar experiment and the lidar data obtained is of marginal utility. A report on this effort is being prepared.

\section{G. Development of Eye-Safe Lasers}

The primary purpose of this part of the project was to enhance LANL's ability to use eye-safe laser technology in support of laser remote sensing projects. This began with an objective evaluation of the merits of all state-of-the-art eyesafe laser technologies that may be applicable to programs being proposed and executed by LANL. Some of these technologies are being pursued adequately elsewhere, and so work on these technologies was limited to a periodically updated evaluation of the literature. Two promising technologies not being adequately pursued elsewhere, "cascade lasing" in erbium materials and development of lasers based on chromium (4+) doped laser materials, were chosen for experimental evaluation and development at LANL under this project. All technologies are compared in a summary technical report that is soon to be released. 


\section{Cascade Lasing}

A significant improvement in the eye-safe $1.6 \mu \mathrm{m}$ laser output of erbium-doped crystals was demonstrated using a novel "cascade laser" cavity design. In this scheme the erbium ions are pumped to high energy levels using $0.97 \mu \mathrm{m}$ commercial laser diodes. In normal erbium lasers, only a small fraction of this energy decays, by phonon processes that heat the crystal, to the initial state of the $1.6 \mu \mathrm{m}$ transition. In the cascade scheme we have adopted a cavity that provides optical feedback at the $2.8 \mu \mathrm{m}$ as well as the $1.6 \mu \mathrm{m}$ wavelength. The $2.8 \mu \mathrm{m}$ laser process channels energy by photon processes to the initial level for $1.6 \mu \mathrm{m}$ lasing. In addition to improving the laser efficiency, this process reduces the very detrimental heating process common in erbium lasers.

In one form of the concept a semi-monolithic laser was fabricated from an $\operatorname{Er}[0.5 \%$ ]:YLF sample $1 \mathrm{~cm}$ in length. One end of the crystal was polished flat and anti-reflection coated for 1.4-1.7 $\mu \mathrm{m}$. The other end of the crystal was polished with a $25-\mathrm{cm}$ convex radius and coated for high reflectivity at $2.72 \mu \mathrm{m}$ and $1 \%$ transmission at $1.59 \mu \mathrm{m}$, forming the output coupler. A 5-cm radius-of-curvature mirror coated for high reflectivity at $1.59 \mu \mathrm{m}$ and $1 \%$ transmission at $2.72 \mu \mathrm{m}$ completes the cavity. This cavity, which induces simultaneous lasing at both $2.8 \mu \mathrm{m}$ and the eye-safe transition at $1.6 \mu \mathrm{m}$, provides considerable improvement in eye-safe laser performance. The slope efficiency of $0.19 \%$ for "normal" erbium lasing is improved to $7 \%$ by this cascade lasing. Rate equation modeling has confirmed that, if losses are reduced, even greater improvement might be obtained. This work, and associated modeling, is summarized in detail in LANL Technical Report LAUR 94-4281. These results have demonstrated excellent feasibilty of developing compact, efficient, eye-safe transmitters. Industrial interest in the technology is high.

\section{Chromium 4+ Materials}

Experimental laser crystals with special charge-compensating dopant combinations that stabilize the tetravalent state of chromium were acquired and evaluated. This was the first objective comparison of Russian and (new) American-grown laser crystals. Chromium ions offer the advantage of tunability in the 1.3 to $1.6 \mu \mathrm{m}$ spectral range. Excellent laser output approaching 1 watt in power was obtained from the best crystals when pumped by a $1 \mu \mathrm{m} \mathrm{Nd:YLF} \mathrm{laser.} \mathrm{Good} \mathrm{improvement} \mathrm{in} \mathrm{material} \mathrm{quality} \mathrm{over}$ previous reports was demonstrated. Feedback to US suppliers has enhanced the quality of their material to approximately match the Russian crystals. This project also included 
comprehensive evaluation of a saturation phenomenon that was considered to limit performance. It was determined that this effect is not as severe during the lasing process as is indicated by the more customary evaluation using fluorescence measurements. A pumpbeam polarization-dependence study has led to a laser design that should couple pump light to the laser transition more effectively. Both diode-laser-pumping and pulsed-Nd:YAG laser-pumping configurations have been designed within the project. A new $\mathrm{Cr} 4+$ crystal $(\mathrm{Cr}, \mathrm{Ca}, \mathrm{GGG})$ was also evaluated and found to have a more desirable output wavelength range but a faster fluorescence lifetime that reduces the projections of achievable laser efficiency and power.

\section{References}

1. Marchbanks, R. D., P. Xie, R. R. Petrin and N. J. Cockroft, "Dual Wavelength Erbium-Doped Cascade Fiber Laser," Paper CMK3, CLEO'94, Anaheim, California, LANL Report LAUR-93-3985.

2. Marchbanks, R. D., R. R. Petrin and N. J. Cockroft, "Improved Performance for the 1590 nm Er:YLF Laser," Paper CTu151, CLEO '95, Baltimore, Maryland, LANL Report LAUR-94-3823.

3. Marchbanks, R. D., "Cascade Lasing in Er:YLF and Er:ZBLAN Fiber," LANL Report LAUR-94-42.

4. Carrig, T. J., R. R. Petrin and N. J. Cockroft, "Spectroscopic Properties of Cr,Ca, GGG," submitted to Advanced Solid State Laser Meeting, 1996, LANL Report LAUR 95-3318.

5. Baker, W. E., G. D. Emmitt, F. Robertson, et. al., "Lidar-Measured Winds from Space: A Key Component for Weather and Climate Prediction," Bulletin of the American Meteorological Society, Vol. 76, no. 6, June 1995 and references cited therein.

6. Sroga, J. T. and E. W. Eloranta,"Lidar Measurement of Wind Velocity Profiles in the Boundary Layer," Journal of Applied Meteorology, Vol. 19, May 1980, p. 598. 